\title{
Dermatomal Distributed Skin Diseases: A Systematic Review
}

\author{
(1) Selin Bayrak¹, (1) Tuğba Kevser Üstünbaș Uzunçakmak², (1) Burhan Engin², (1) Server Serdaroğlu²
}

${ }^{1}$ Azerbaijan Medical University Faculty of Medicine, Department of Dermatology, Baku, Azerbaijan

2Istanbul University Cerrahpasa-Cerrahpasa Faculty of Medicine, Department of Dermatology, Istanbul, Turkey

\section{ABSTRACT}

Dermatomal distribution of cutaneous lesions is a common pattern that encountered in the field of dermatology. Being familiar with these types of patterns can help evaluating the specifically distributed lesions and may shed a light on the diagnosis. This review summarizes the literature about dermatoses that presents with dermatomal distribution.

Keywords: Blaschko, Dermatology, Dermatome, Herpes zoster, Lichen planus, Melanoma, Metastasis, Psoriasis

\section{Introduction}

Recognition of patterns is essential assessing the skin diseases. The skin presents a distinct anatomical pattern due to distribution of sensory nerve fibers originate from a single spinal nerve [1]. An area of skin supplied by a single nerve root is called a dermatome. It can be considered as communicating sensation from skin to the brain. Dermatomes are located horizontally on the trunk and this horizontal pattern contrasts with the pattern of the extremities where it is typically longitudinal. Dermatomes can overlap and an individual's exact pattern of dermatome is unique [2].

A zosteriform pattern describes a unilateral belt like or girdle like presentation of a dermatosis on the area of skin that innervated by sensory branch of a spinal nerve [3-5].

While herpes zoster $(\mathrm{HZ})$ is first to remember between zosteriform dermatoses, a large variety of inflammatory, infectious, neoplastic diseases may also present as zosteriform lesions [6].

There are 8 pairs of cervical, 12 pairs of thoracic, 5 pairs lumbar, 5 pairs of sacral and a pair of coccygeal nerves. There are 30 different dermatomes, despite the fact there are 31 pairs of spinal nerves. This results from the fact that C1 spinal nerve typically doesn't have sensory fibers. As a result, dermatomes start from spinal nerve C2.
Even though skin mostly innervated by spinal nerves, the facial skin innervated by cranial nerve $V$ which known as trigeminal nerve [6].

\section{Pathogenesis}

Cutaneous diseases that present itself with dermatomal distributed lesions reflects mainly 2 mechanisms.

The isotopic mechanism;

Isotopic response defined as a dermatosis that located and remains restricted to a site; which is the same area that other previously healed skin disease has placed [7-9].

The archetype of this phenomenon is a dermatosis that occurring on the same site of recovered previous $\mathrm{HZ}$.

Although the precise mechanism of isotopic response needs to be enlighten it may be related to varicella zoster virus (VZV) induced modifications of the cutaneous immune system [10].

The neural mechanism;

Neural pathway results from reactivation of latent VZV of neural cells and the cells of dorsal root ganglia. Newly synthesized viral particles transferred to the skin via microtubular system of the axons [11]. 


\section{Lines of Blaschko}

During embryogenesis, precursor cells start to proliferate on the midline and grow in transversal direction from this line. As the growth progresses these cells set up in $\mathrm{S}$ shape in the back and $\mathrm{V}$ shape on anterolateral trunk. These lines illustrate ectodermal development patterns. This distribution pattern, especially on the trunk, is frequently hard to distinguish from dermatomes [12].

\section{Herpes Zoster}

VZV infection is an important medical entity that concerns many specialties besides dermatology such as infectious diseases and neurology. It can effect variety of individuals from childhood to elderly. Its treatment also requires proficiency in pain management. Varicella generates from acute viremia. HZ appears in later life due to the dormant viral infection. When it is re-activated and virus spreads orthodromically to the target tissue. Generally, one dermatome is involved, however two or three adjoining dermatomes can be effected. The lesions commonly located unilaterally and do not intersect the midline (Figure 1, 2) [13].

If the zosteriform eruption is recent ( $<10-14$ days) $\mathrm{HZ}$ is the most plausible diagnosis. However, zosteriform HSV infections are experienced in nearly $25 \%$ of the cases diagnosed as $\mathrm{HZ}$ at first [14-26]. The differantiation between HSV and VZV can quickly be performed on a Tzanck smear [4]. This difference is important in terms of dosing regimen which is differing from one another [4].

\section{Zosteriform Cutaneous Metastases}

Cutaneous skin metastasis results from tumor cells spreading directly and through hematogenous or lymphatics. It is comorbid in $0.7-10 \%$ of patients with cancer and composes $2 \%$ of cutaneous malignancies. Zosteriform pattern is a rarely seen subtype and there have been less than a hundred case reported [27].

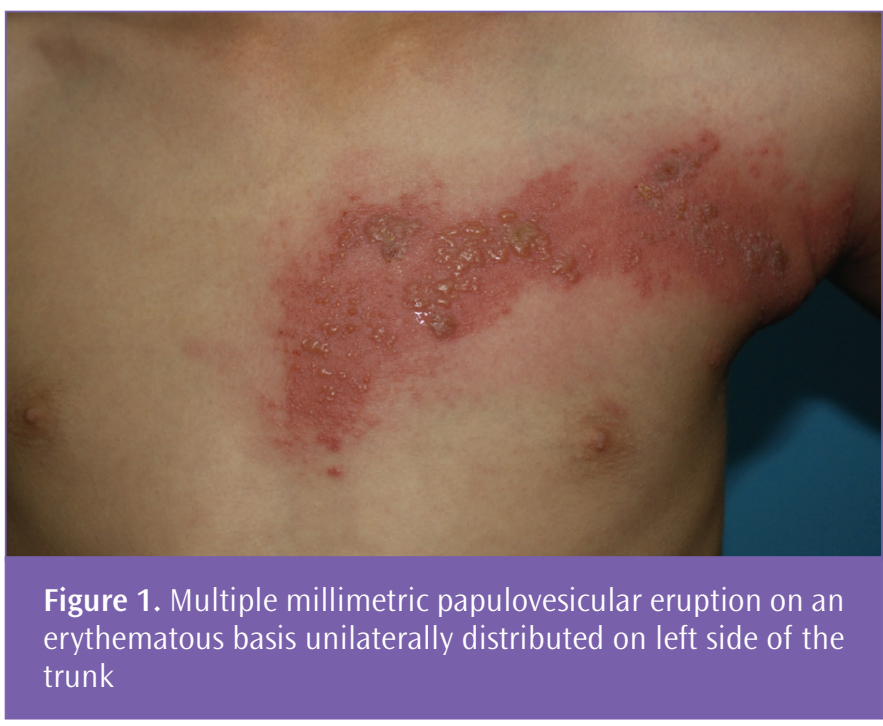

Diagnosis of dermatomal distributed cutaneous metastasis can be tough. It can also prompt untoward investigations, delayed diagnosis and inaccurate treatment.

This sort of distribution pattern has been reported with digestive, respiratory tumors, breast cancer and lymphomas and squamous cell carcinomas (SCC). And the most common sites of metastases were chest and abdominal wall.

Besides the similar location and distribution pattern, patients also reported concomitant pain and that sort of presentation usually leads the physician to $\mathrm{HZ}$ and results in administration of antivirals in many cases [28].

Zosteriform cutaneous metastases generating from internal malignancies have also reported. Most frequently in the older patients, lesions with abnormally long duration as well as in patients with a history of cancer.

Cutaneous metastases of breast cancer are the most frequently observed, presumably due to its primary location on the thoracic dermatomes [29-53]. These zosteriform cutaneous metastases may often be initially misdiagnosed as $\mathrm{HZ}[34,54,55]$ or as contact eczema [56]. And they are frequently a sign of a poor prognosis and cutaneous metastases have been reported in $0.7-9 \%$ of the cancer patients [57]. Although zosteriform distribution of metastases are rare and less known there are few cases reported in the literature.

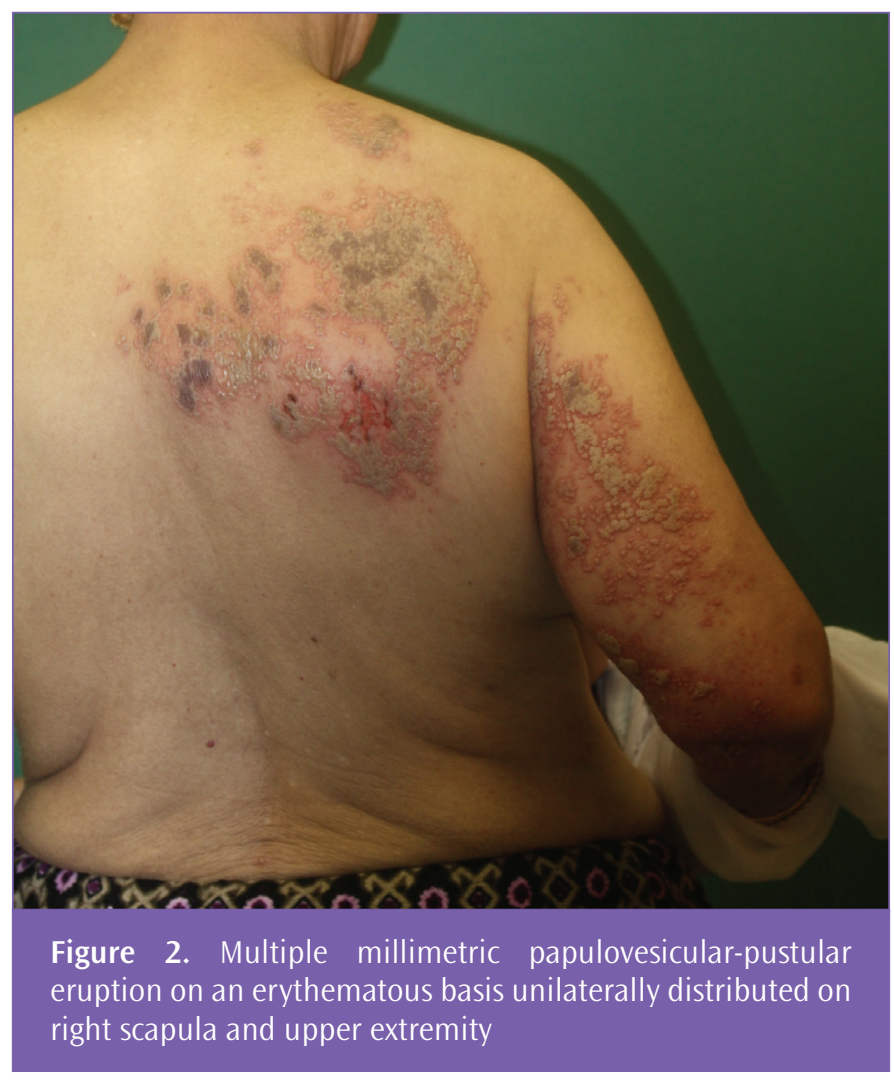




\section{Zosteriform Skin Cancers}

If the eruption is more long established (more than 14 days), zosteriform distributed primary skin cancers should be considered, predominantly SCC but also angiosarcoma, Kaposi's sarcoma, primary cutaneous B-cell lymphoma and primary cutaneous T-cell lymphoma should be suspected [58-66].

\section{Multiple Eccrine Spiradenomas (ES)}

ES is a benign, dermal tumor originating from eccrine sweat glands. It typically presents as a solitary painful lesion, but rarely presents as multiple ES. Multiple ES is estimated to involve less than $2 \%$ of total number of cases. And it has increased incidence in females. The etiology of multiple ES is unknown. This diagnosis is aligned with an increased risk of a malignant transformation. Multiple ES can present in a segmental, linear, blaschkoid, or zosteriform pattern [67].

\section{Mycosis Fungoides (MF)}

MF presents itself with patches and plaques in the sun protected areas. Variety of clinical forms have been reported including granulomatous MF, hypopigmented MF, folliculotropic MF, pagetoid reticulosis.

Williams et al. [68] reported a case of MF mimicking HZ but immunophenotyping was not performed. In 2017 Rieger et al. [69] reported immunophenotypically and molecularly confirmed zosteriform MF.

\section{Lichen Planus}

Lichen planus is an inflammtory mucocutaneous disorder which is characterized by lichenoid, pruritic, shiny, flat papules. In addition to the classical appearance, there are varieties of clinical forms described such as zosteriform lesions. This type of lesions may present zosteriform distribution spontaneously or as Wolf's isotopic response [70-73]. Due to its distribution pattern, it can be also named as linear lichen planus in the literature. Although this rare pattern usually seen as a single dermatomal involvement, occasionally it may represent a multiple dermatomal involvement $[74,75]$.

\section{Melanoma}

Zosteriform metastasis is usually painful or pruritic, and is frequently located on a single dermatome, leaving an open door for a potential misdiagnosis [76].

There are six relevant cases reported about recurrence of melanoma in a zosteriform distribution after treatment with chemotherapy [76-81].

\section{Psoriasis}

Psoriasis is common, chronic relapsing and remitting inflammatory disease with an overall prevalence of $2 \%$ to $3 \%$ of the world's population. Koebner phenomenon (KP), also called as isomorphic response, is referred to the occurance of psoriasiform lesions after trauma on healthy skin sites in psoriatic patients. About $25 \%$ of the psoriasis patients develop KP after various traumatic injuries. $\mathrm{KP}$ lesions are always located at the site of pre-existed $\mathrm{HZ}$ eruption with a latent period of approximately one week to four months from the occurrence of $\mathrm{HZ}$ [82]. There are one cases reported in literature each resulting from KP and Wolf isotopic response $[82,83]$.

\section{Other Skin Diseases}

Additional skin diseases that may appear as zosteriform pattern include zosteriform perforating collagenosis, unilateral nevoid telangiectasia, zosteriform nevus spilus with melanoma, transient acantholytic dermatosis, progressive cribriform and zosteriform hyperpigmentation, Spitz nevi, epitheloid hemangioma, porokeratosis zosteriform nevus spilus, arterio-venous malformations, and segmental vitiligo may also present with a zosteriform pattern [84-98]. Although there is another publication about segmental vitiligo being a misnomer and rather than a dermatomal distributed disease it may be results from cutaneous mosaism [99].

Zosteriform inflammatory lesions may appear as drug reactions to levofloxacin [100]. Uncommon cases are accounted for zosteriform morphea [101]. Incidental cases of zosteriform leishmaniosis have been described [102-105].

\section{Conclusion}

Dermatomal distribution instantly reminds physicians about $\mathrm{HZ}$. Considering the patients age, competence of their immune system, history of previous dermatoses located in the same area and other relevant diseases such as cancer should be taken into account in order to avoid unnecessary use of antivirals and incorrect diagnoses. Zosteriform cutaneous metastases are not frequent but they are being reported in the literature as a consequence of large variety of cancers.

\section{Ethics}

Peer-review: Internally peer-reviewed.

\section{Authorship Contributions}

Surgical and Medical Practices: S.B., T.K.Ü.U., Concept: S.B., T.K.Ü.U., B.E., S.S., Design: S.B., T.K.Ü.U., B.E., S.S., Data Collection or Processing: S.B., B.E., Analysis or Interpretation: S.B., T.K.Ü.U., B.E., S.S., Literature Search: S.B., Writing: S.B., T.K.Ü.U., S.S. 
Conflict of Interest: No conflict of interest was declared by the authors.

Financial Disclosure: The authors declared that this study received no financial support.

\section{References}

1. Tan TC, Black PM. The contributions of Otfrid Foerster (1873-1941) to neurology and neurosurgery. Neurosurgery 2001;49:1231-1236.

2. Whitman PA, Adigun 00. Anatomy, Skin, Dermatomes. In: StatPearls Treasure Island (FL): StatPearls Publishing; December 10, 2018.

3. Arvin AM. Varicella-zoster virus: overview and clinical manifestations. Semin Dermatol 1996;15(2 Suppl 1):4-7.

4. Nikkels AF, Piérard GE. Oral antivirals revisited in the treatment of herpes zoster: what do they accomplish? Am J Clin Dermatol 2002;3:591-598.

5. Mueller NH, Gilden DH, Cohrs RJ, Mahalingam R, Nagel MA. Varicella zoster virus infection: clinical features, molecular pathogenesis of disease, and latency. Neurol Clin 2008;26:675-697.

6. Ludwig PE, Reddy V, Varacallo M. Neuroanatomy, Central Nervous System (CNS). Updated: 2020 May 24. In: StatPearls [Internet]. Treasure Island (FL): StatPearls Publishing; 2020.

7. Kroth J, Tischer J, Samtleben W, Weiss C, Ruzicka T, Wollenberg A. Isotopic response, Köbner phenomenon and Renbök phenomenon following herpes zoster. J Dermatol 2011;38:1058-1061.

8. Wolf R, Brenner S, Ruocco V, Filioli FG. Isotopic response. Int J Dermatol 1995;34:341-348.

9. Wolf R, Wolf D, Ruocco E, Brunetti G, Ruocco V. Wolf's isotopic response. Clin Dermatol 2011;29:237-240.

10. Twersky JM, Nordlund JJ. Cutaneous T-cell lymphoma sparing resolving dermatomal herpes zoster lesions: an unusual phenomenon and implications for pathophysiology. J Am Acad Dermatol 2004;51:123-126.

11. Castronovo C, Nikkels AF. Chronic herpes zoster duplex bilateralis. Acta Derm Venereol 2012;92:148-151.

12. Kouzak SS, Mendes MS, Costa IM. Cutaneous mosaicisms: concepts, patterns and classifications. An Bras Dermatol 2013:88:507-517.

13. Dayan RR, Peleg R. Herpes zoster - typical and atypical presentations. Postgrad Med 2017;129:567-571.

14. Takayama N, Takayama M, Takita J. Herpes simplex mimicking herpes zoster in a child immunized with varicella vaccine. Pediatr Infect Dis J 2001;20:226-228.

15. Summers BC, Margolis TP, Leib DA. Herpes simplex virus type 1 corneal infection results in periocular disease by zosteriform spread. J Virol 2001;75:5069-5075.

16. Koh M], Seah PP, Teo RY. Zosteriform herpes simplex. Singapore Med 2008;49:59-60.

17. Aithal S, Kuruvila S, Ganguly S. Zosteriform herpes simplex and herpes zoster: A clinical clue. Indian Dermatol Online J 2013;4:369.

18. Saito K, Hatano Y, Sakai T, Tatewaki S, Sako Y, Fujiwara S. Primary ocular herpes simplex virus infection with zosteriform spreading, accompanied with meningism. J Dermatol 2012;39:489-490.

19. Udayashankar C, Oudeacoumar P, Nath AK. Recurrence of zosteriform lesions on the contralateral dermatome: A diagnostic dilemma. Indian Dermatol Online J 2012;3:76-77.

20. Goel N, Mao H, Rong Q, Docherty JJ, Zimmerman D, Rosenthal KS. The ability of an HSV strain to initiate zosteriform spread correlates with its neuroinvasive disease potential. Arch Virol 2002;147:763-773.
21. Rübben A, Baron JM, Grussendorf-Conen El. Routine detection of herpes simplex virus and varicella zoster virus by polymerase chain reaction reveals that initial herpes zoster is frequently misdiagnosed as herpes simplex. $\mathrm{Br}$ ] Dermatol 1997;137:259-261.

22. Yamamoto S, Shimomura Y, Kinoshita S, Tano Y. Differentiating zosteriform herpes simplex from ophthalmic zoster. Arch Ophthalmol 1994;112:15151516.

23. Ford RA. Zosteriform eruption of herpes simplex virus after vertebral metastases. Cutis 1993;52:99-100.

24. Groisser D, Taylor S, Grossman ME. Postsurgical zosteriform herpes simplex 2 in noncontiguous dermatomes. J Am Acad Dermatol 1990;23:928-930.

25. Kalman CM, Laskin OL. Herpes zoster and zosteriform herpes simplex virus infections in immunocompetent adults. Am J Med 1986;81:775-778.

26. Forrest WM, Kaufman HE. Zosteriform herpes simplex. Am J Ophthalmol 1976;81:86-88

27. Dagdelen D, Karadag AS, Akdeniz N, Ozkanli SS, Gurel MS. Zosteriform cutaneous metastasis: A case series. Dermatol Ther 2020:e14137.

28. Thomaidou E, Armoni G, Klapholz, L, Hadayer N, Maly A, Ramot Y. Zosteriform cutaneous metastases. Clin Exp Dermatol 2018;43:734-736.

29. Bluefarb SM, Wallk S, Gecht M. Carcinoma of the prostate with zosteriform cutaneous lesions. AMA Arch Derm 1957;76:402-407.

30. Manteaux A, Cohen PR, Rapini RP. Zosteriform and epidermotropic metastasis. Report of two cases. J Dermatol Surg Oncol 1992;18:97-100.

31. New WN. Leiomyoma cutis (zosteriform). Arch Derm Syphilol 1949;60:461.

32. Hodge SJ, Mackel S, Owen LG. Zosteriform inflammatory metastatic carcinoma. Int J Dermatol 1979;18:142-145.

33. Wakelin SH, Young E, Kelly S, Turner M. Transient leukaemia cutis in chronic lymphocytic leukaemia. Clin Exp Dermatol 1997;22:37-40.

34. Kishan KH, Rao G. A rare case of zosteriform cutaneous metastases from squamous cell carcinoma of hard palate. Ann Med Health Sci Res 2013;3:127130

35. Heckmann M, Volkenandt M, Lengyel ER, Schirren CG, Gizycki-Nienhaus BV. Cytological diagnosis of zosteriform skin metastases in undiagnosed breast carcinoma. Br J Dermatol 1996;135:502-503.

36. Cecchi R, Brunetti L, Bartoli L, Pavesi M, Giomi A. Zosteriform skin metastases from breast carcinoma in association with herpes zoster. Int J Dermatol 1998;37:476-477.

37. Matarasso SL, Rosen T. Zosteriform metastasis: case presentation and review of the literature. J Dermatol Surg Oncol 1988;14:774-778.

38. Arfan-ul-Bari. Zosteriform cutaneous leiomyoma: a rare cutaneous neoplasm. J Coll Physicians Surg Pak 2013;23:586-587.

39. Zucchi A, Aimi F, Cardis A, Santini M, Tognetti E, Zambito-Spadaro F, De Panfilis G, Fabrizi G. Unusual form of cutaneous infiltration by cancer. Acta Biomed 2012;83:51-52.

40. Trojjet S, Hammami H, Zaraa I, Bouzguarrou A, Joens M, Haouet S, Ben Osman A, Mokni M. Chronic lymphocytic leukemia revealed by a branulomatous zosteriform eruption. Skinmed 2012;10:50-52.

41. Li WH, Tu CY, Hsieh TC, Wu PY. Zosteriform skin metastasis of lung cancer Chest 2012;142:1652-1654.

42. Hapgood G, Mooney E, Dinh HV, Gin D, McLean C, Ting SB. Leukaemia cutis in chronic lymphocytic leukaemia following varicella zoster virus reactivation. Intern Med J 2012;42:1355-1358.

43. González-Hernández S, Pérez-Robayna N, Rodríguez-García C, Sánchez González R. [Cutaneous metastases with zosteriform pattern from a breast carcinoma]. Med Clin (Barc) 2012;138:460. 
44. Cecchi R, Fancelli L, Troiano M. Melanoma arising in giant zosteriform nevus spilus. Indian J Dermatol Venereol Leprol 2012;78:643-645.

45. Chandanwale SS, Gore CR, Buch AC, Misal SS. Zosteriform cutaneous metastasis: a primary manifestation of carcinoma breast, rare case report. Indian J Pathol Microbiol 2011;54:863-864.

46. Virmani NC, Sharma YK, Panicker NK, Dash KN, Patvekar MA, Deo KS. Zosteriform skin metastases: clue to an undiagnosed breast cancer. Indian J Dermatol 2011;56:726-727.

47. Dincer D, Tunca M, Akar A, Kurt B. Zosteriform cutaneous metastasis of renal cell carcinoma. Indian J Dermatol Venereol Leprol 2011;77:340-342.

48. Eichinger J, George B, Myhand R. Cutaneous metastatic rectal carcinoma masquerading as herpes zoster. South Med J 2011;104:233-235.

49. Augustin G, Kekez T, Bogdanic B. Abdominal papular zosteriform cutaneous metastases from endometrial adenocarcinoma. Int J Gynaecol Obstet 2010;110:74.

50. Al Zouman A, Al Harthi F. Male breast carcinoma with zosteriform metastasis. Breast J 2010;16:88-89.

51. Monteagudo Sánchez B, Cabanillas González M, Pérez Fernández A, de la Cámara Gómez J, Campo Cerecedo F. Zosteriform cutaneous metastases of malignant pleural mesothelioma. Rev Clin Esp 2009;209:262-263.

52. Claeys A, Pouaha J, Christian B, Froment N, Truchetet F. Zosteriform cutaneous localizations of B-cell chronic lymphocytic leukaemia. Eur J Dermatol 2008;18:101-102.

53. Rasi A, Tajziehchi L, Shaianfar N. Metastatic carcinoma of the ovary presenting as zosteriform lesions. Arch Iran Med 2007;10:250-252.

54. LeSueur BW, Abraham RJ, DiCaudo DJ, O'Connor WJ. Zosteriform skin metastases. Int J Dermatol 2004;43:126-128.

55. Williams LR, Levine LJ, Kauh YC. Cutaneous malignancies mimicking herpes zoster. Int J Dermatol 1991;30:432-434.

56. Eichinger J, George B, Myhand R. Cutaneous metastatic rectal carcinoma masquerading as herpes zoster. South Med J 2011;104:233-235

57. Rao R, Balachandran C, Rao L. Zosteriform cutaneous metastases: A case report and brief review of literature. Indian J Dermatol Venereol Leprol 2010;76:447.

58. Kast DR, Sammons D, Nixon RMS, Geiss DF. Cutaneous angiosarcoma masquerading as herpes zoster. Cutis 2012;90:42-45.

59. Kishan KH, Rao Grr. A rare case of zosteriform cutaneous metastases from squamous cell carcinoma of hard palate. Ann Med Health Sci Res 2013;3:127130.

60. Minakawa S, Nakajima K, Aizu T, Kaimori M, Nomura K. A case of zosteriform metastatic skin cancer. Clin Exp Dermatol 2008;33:808-810.

61. Cohen JL, Barankin B, Zloty DM, Mikhail GR. Metastatic zosteriform squamous cell carcinoma in an immunocompetent patient. J Cutan Med Surg 2004;8:438-441.

62. Watabe H, Kawakami T, Soma Y, Baba T, Mizoguchi M. Primary cutaneous T-cell-rich B-cell lymphoma in a zosteriform distribution associated with Epstein-Barr virus infection. J Dermatol 2002;29:748-753.

63. Bauzá A, Redondo P, Idoate MA. Cutaneous zosteriform squamous cell carcinoma metastasis arising in an immunocompetent patient. Clin Exp Dermatol 2002;27:199-201.

64. Shafqat A, Viehman GE, Myers SA. Cutaneous squamous cell carcinoma with zosteriform metastasis in a transplant recipient. J Am Acad Dermato 1997;37:1008-1009.

65. Eisman S, O'Toole EA, Collis C, Rustin MH. Zosteriform Kaposi's sarcoma. Clin Exp Dermatol 2001;26:402-404.
66. Ricci RM, Latham PL, Soong V, Mullins D. Zosteriform cutaneous T-cell lymphoma. J Am Acad Dermatol 1995;32:127-128.

67. Ren F, Hu Z, Kong Q, Sang H. Multiple Segmental Eccrine Spiradenoma with a Zosteriform Pattern: A Case Report and Literature Review. Ann Dermatol 2015;27:435-438.

68. Williams LR, Levine LJ, Kauh YC. Cutaneous Malignancies Mimicking Herpes Zoster. Int J Dermatol 1991;30:432-434.

69. Rieger KE, Kim J, Kim YH. Zosteriform Mycosis Fungoides: A New Clinical Presentation With a Dermatomal Distribution. Am J Dermatopathol 2017;39:17-18.

70. Türel A, Oztürkcan S, Sahin MT, Türkdoğan P. Wolf's Isotopic Response: A Case of Zosteriform Lichen Planus. J Dermatol 2002;29:339-342.

71. Gajjar PC, Mehta HH, Nimbark V, Jethwa M. An atypical clinical presentation of lichen planus pigmentosus with typical dermoscopic pattern. Australas J Dermatol 2018;59:e208-e210.

72. Ganguly S, Jaykar KC. Twenty nail dystrophy in association with zosteriform lichen planus. Indian J Dermatol 2012;57:329.

73. Perry D, Fazel N. Zosteriform lichen planus. Dermatol Online J 2006;12:3.

74. Manjunath S, Kamat D, Kumaran MS. Multidermatomal zosteriform lichen planus pigmentosus-a unique presentation. Int J Dermatol 2019;58:343-344.

75. Jivani N, Kota RS, Nair PA.Unilateral zosteriform lichen planus involving multiple noncontiguous dermatomes with oral involvement. Indian Dermatol Online J 2016;7:539-541.

76. Ibrahim S, Al-Turk B, Harris C, Al-Saffar F, Said S, Farsi M, Winder J, Landa C. Melanoma Masquerading as a Zosteriform Rash. Am J Case Rep 2017;18:537-540.

77. Sinha R, Fearfield L. Images in clinical medicine. Zosteriform metastases from malignant melanoma. N Engl J Med 2014;370:22.

78. Kondras K, Zalewska A, Janowski P, Kordek R. Cutaneous multifocal melanoma metastases clinically resembling herpes zoster. J Eur Acad Dermatol Venereol 2006;20:470-472.

79. Bergler-Czop B, Brzezinska-Wcislo L, Miziolek B. Metastases of malignant melanoma to the skin of abdomen and thorax imitating Herpes Zoster infection. Ann Saudi Med 2015;35:482-483.

80. Savoia P, Fava P, Deboli T, Quaglino P, Bernengo MG. Zosteriform cutaneous metastases: a literature meta-analysis and a clinical report of three melanoma cases. Dermatol Surg 2009;35:1355-1363.

81. Evans AV, Child FJ, Russell-Jones R. Zosteriform metastasis from melanoma BMJ 2003;326:1025-1026

82. Zhao YK, Zhang YQ, Wang F, Wu HH, Luo ZY, Luo DQ, Chen WN. Developing Shingles-Induced Koebner Phenomenon in a Patient With Psoriasis: A Case Report. Medicine (Baltimore) 2015;94:e1009.

83. Garcia-Souto F, Martínez-Barranca ML, Pérez-Gil A, Escudero-Ordoñez J. Visual Dermatology: Wolf's Isotopic Response: Zosteriform Psoriasis. J Cutan Med Surg 2019;23:462.

84. Nakanishi G, Tsunemitsu R, Akagi O. Reactive perforating collagenosis occurring in a zosteriform distribution. Br J Dermatol 1999;141:367-369.

85. Senel E, Güleç AT. Congenital unilateral naevoid telangiectasia with a wide dermatomal distribution. Clin Exp Dermatol 2008;33:663-664.

86. Wilkin JK, Smith JG Jr, Cullison DA, Peters GE, Rodriquez-Rigau LJ, Feucht CL. Unilateral dermatomal superficial telangiectasia. Nine new cases and a review of unilateral dermatomal superficial telangiectasia. J Am Acad Dermatol 1983;8:468-477.

87. Liss WA, Norins AL. Zosteriform transient acantholytic dermatosis. J Am Acad Dermatol 1993;29:797-798. 
88. Lee HJ, Jung KE, Kim HS, Lee JY, Kim HO, Park YM. Zosteriform transient acantholytic dermatosis. J Dermatol 2014;41:104-105.

89. Gutte RM. Progressive cribriform and zosteriform hyperpigmentation. Indian Dermatol Online J 2014;5:38-40.

90. Goyal T. Progressive cribriform and zosteriform hyperpigmentation: Where are we at present? Indian Dermatol Online J 2013;4:264-266.

91. Glasgow MA, Lain EL, Kincannon JM. Agminated Spitz nevi: report of a child with a unique dermatomal distribution. Pediatr Dermatol 2005;22:546-549.

92. Kurihara $\mathrm{Y}$, Inoue $\mathrm{H}$, Kiryu $H$, Furue M. Epithelioid hemangioma (angiolymphoid hyperplasia with eosinophilia) in zosteriform distribution. Indian J Dermatol 2012;57:401-403.

93. Agrawal SK, Gandhi V, Madan V, Bhattacharya SN. Topical tretinoin in Indian male with zosteriform porokeratosis. Int J Dermatol 2003;42:919-920.

94. Hu N, Zhong J, Yang B, Wang L, Li L. A case of Zosteriform nevus spilus with halo nevus. Int J Dermatol 2013;52:386-388.

95. Yeh SW, Magalhaes AM, Vasconcellos MR, Michalany NS, Tomimori Yamashita J. Zosteriform connective tissue nevus: a case report. Int J Dermatol 2003;42:720-722.

96. Choi YJ, Lee SJ, Choi CW, Kim WS, Lee GY. Multiple unilateral zosteriform connective tissue nevi on the trunk. Ann Dermatol 2011;23(Suppl 2):243-246.
97. Lee JY, Ok SJ, Oh CK, Park SK, Kim DW, Yang JY. Spinal arteriovenous malformation masquerating zoster sine herpete. Korean J Pain 2013;26:7275.

98. Choltz JR, Williamson C. Vitiligo in apparent dermatomal distribution. AMA Arch Derm Syphilol 1951;64:366-369.

99. van Geel N, Speeckaert R. Segmental Vitiligo. Dermatol Clin 2017;35:145-150.

100. Vetrichevvel TP, Sudha R, Shobana S, Anandan S. Zosteriform fixed drug eruption to levofloxacin. Indian J Dermatol 2012;57:327-328.

101. Ruiz-Villaverde R, Sánchez-Cano D. Zosteriform morphea in an immunocompetent patient. Indian J Dermatol 2013;58:411.

102. Omidian M, Mapar MA. Chronic zosteriform cutaneous leishmaniasis. Indian J Dermatol Venereol Leprol 2006;72:41-42.

103. Ceyhan AM, Yildirim M, Basak PY, Akkaya VB, Erturan I. A case of erysipeloid cutaneous leishmaniasis: atypical and unusual clinical variant. Am J Trop Med Hyg 2008;78:406-408.

104. Bari AU, Rahman SB. Many faces of cutaneous leishmaniasis. Indian J Dermatol Venereol Leprol 2008;74:23-27.

105. Bongiorno MR, Pistone G, Aricò M. Unusual clinical variants of cutaneous leishmaniasis in Sicily. Int J Dermatol 2009;48:286-289. 